\title{
ON FREE GROUP ALGEBRAS IN DIVISION RINGS WITH UNCOUNTABLE CENTER
}

\author{
J. GONCALVES AND M. SHIRVANI
}

(Communicated by Ken Goodearl)

\begin{abstract}
Let $D$ be a division algebra with uncountable center $k$. If $D$ contains a noncommutative free $k$-algebra, then $D$ also contains the $k$-group algebra of the free group of rank 2.
\end{abstract}

\section{INTRODUCTION}

Let $D$ be a division ring with center $k$. In [7] Makar-Limanov states the following conjecture:

(ML) If $D$ is finitely-generated and infinite dimensional over $k$, then $D$ contains a 2-generator free algebra.

An earlier claim about the existence of the free subgroups in division rings was stated by Lichtman [2]:

(L) The multiplicative group of a noncommutative division ring contains a free group of rank 2.

Evidence for (ML) can be found in [1], [5], [11], [12], and for (L) in [2], [3]. Recently, Makar-Limanov [11] surprisingly proved that the ring of fractions of the first Weyl algebra $A_{1}$, over the complex field $\mathbb{C}$, contains a $\mathbb{C}$-free group algebra (= the group algebra of a free group over $\mathbb{C}$ ).

In this note we show that this result is not accidental, but a general phenomenon. We will use a reasoning similar to the one in [9] to prove:

Theorem. If $k$ is uncountable and $D$ contains a $k$-free algebra of rank 2 , then $D$ contains a $k$-free group algebra.

Thus, we obtain new examples of division rings containing free group algebras. In particular, if $k$ is uncountable, then (ML) implies (L).

We observe that $A_{1}$ can be regarded as a skew polynomial ring over the field of rational functions $\mathbb{C}(t)$. Therefore the existence of free group algebras in the ring of fractions of $A_{1}$ follows from the existence of free algebras in the ring of fractions of skew polynomial rings. (See [1], [5] for more on this.)

\section{Proof of the theOrem}

The lemma below is proved in [12], Lemma 1, for algebras inside domains. It is also true for free group algebras.

Received by the editors September 21, 1993 and, in revised form, July 25, 1994.

1991 Mathematics Subject Classification. Primary 16A39; Secondary 16A06, 16A48.

Key words and phrases. Division ring, free algebra, free group algebra. 
Lemma 2.1. Let $A$ be a domain with prime subfield $k_{0}$, and let $k$ be any central subfield of $A$. If $x_{1}, x_{2} \in A$, then $x_{1}$ and $x_{2}$ are free over $k_{0}$ if and only if they are free over $k$.

We also need the following:

Lemma 2.2. Let $D[x]$ be the polynomial ring in the central indeterminate $x$, and let $D(x)$ be its ring of fractions. If $f \in D(x)$ and $f(z)=0$ for infinitely many $z \in k$, then $f$ is identically zero.

Proof. See [9], Lemma 1.

Let $L$ be a field, and let us denote by $L\left\{x_{1}, x_{2}\right\}$ the free $L$-group algebra on the generators $x_{1}$ and $x_{2}$. Let $L\left\langle y_{1}, y_{2}\right\rangle$ be the free $L$-algebra in the generators $y_{1}$ and $y_{2}$, and let $L\left\langle y_{1}, y_{2}\right\rangle\left[\left[\lambda_{1}, \lambda_{2}\right]\right]$ be the ring of formal power series in the central indeterminates $\lambda_{1}$ and $\lambda_{2}$ over $L\left\langle y_{1}, y_{2}\right\rangle$. With this notation we have:

Lemma 2.3. The homomorphism $\psi$ from $L\left\{x_{1}, x_{2}\right\}$ to $L\left\langle y_{1}, y_{2}\right\rangle\left[\left[\lambda_{1}, \lambda_{2}\right]\right]$ defined by

$$
\psi\left(x_{i}\right)=1+\lambda_{i} y_{i}, \quad \psi\left(x_{i}^{-1}\right)=\sum_{j=0}^{\infty}\left(-\lambda_{i} y_{i}\right)^{j}, \quad i=1,2
$$

is injective.

Proof. See [10], Lemma 1.

Proof of the theorem. Let $a$ and $b$ be elements of $D$ that generate a free $k$-algebra. We claim that there exist $\sigma$ and $\tau$ in $k$ such that $1+\sigma a$ and $1+\tau b$ generate a free $k$ group algebra. In view of Lemma 2.1 it is enough to show that they generate a free $k_{0}$-group algebra. Fix $\sigma \neq-a^{-1}$. Since $k_{0}\left\{x_{1}, x_{2}\right\}$ is countable but $k$ is not, there exists $0 \neq f_{\sigma}\left(x_{1}, x_{2}\right) \in k_{0}\left\{x_{1}, x_{2}\right\}$ such that for uncountably many $\tau \in k \backslash\left\{-b^{-1}\right\}$ we have

$$
f_{\sigma}(1+\sigma a, 1+\tau b)=0
$$

By Lemma 2.2 we may replace $\tau$ by the central variable $\lambda_{2}$, obtaining the identity

$$
f_{\sigma}\left(1+\sigma a, 1+\lambda_{2} b\right)=0 \quad \text { in } D\left(\lambda_{2}\right) .
$$

Arguing analogously, we may find a $0 \neq f\left(x_{1}, x_{2}\right) \in k_{0}\left\{x_{1}, x_{2}\right\}$ among all $f_{\sigma}$, such that

$$
f\left(1+\lambda_{1} a, 1+\lambda_{2} b\right)=0 \quad \text { in } D\left(\lambda_{2}\right)\left(\lambda_{1}\right) .
$$

Now we embed $D\left(\lambda_{1}\right)\left(\lambda_{2}\right)$ in the Laurent series division ring $D\left(\left(\lambda_{2}\right)\right)\left(\left(\lambda_{1}\right)\right)$. We obtain

$$
0 \neq \psi\left(f\left(x_{1}, x_{2}\right)\right)=\lambda_{1}^{m_{1}} \lambda_{2}^{m_{2}} f_{m_{1}, m_{2}}\left(y_{1}, y_{2}\right)+R,
$$

with $f_{m_{1}, m_{2}} \neq 0$, and where all the terms in $R$ possess higher $\left(\lambda_{1}, \lambda_{2}\right)$-degree (in the lexicographic order, say). But from $(*) f_{m_{1}, m_{2}}(a, b)=0$. This contradicts the hypothesis that $a$ and $b$ generate a free $k_{0}$-algebra. The result follows. 


\section{REFERENCES}

1. J. Z. Goncalves and M. Shirvani, On free subalgebras in division algebras and related rings, preprint.

2. A. I. Lichtman, On subgroups of the multiplicative group of skew fields, Proc. Amer. Math. Soc. 63 (1977), 15-16. MR 56:5744

3. - Free subgroups of normal subgroups of the multiplicative group of skew fields, Proc. Amer. Math. Soc. 71 (1978), 174-178. MR 58:779

4. _ On normal subgroups of the multiplicative group of skew fields generated by a polycyclic-by-finite group, J. Algebra 78 (1982), 548-577. MR 84f: 16016

5. M. Lorenz, On free subalgebras of certain division algebras, Proc. Amer. Math. Soc. 98 (1986), 401-405. MR 87m:16040

6. L. Makar-Limanov, The skew field of fractions of the Weyl algebra contains a free noncommutative subalgebra, Comm. Algebra 17 (1983), 2003-2006. MR 84j:16012

7. _ On free subobjects of skew fields, Methods in Ring Theory (F. van Oystaeyen, ed.), Reidel, Dordrecht, 1984, pp. 281-285. CMP 17:06

8. _ On group rings of nilpotent groups, Israel J. Math. 48 (1984), 245-248. MR 86c: 16006

9. L. Makar-Limanov and P. Malcolmson, Words periodic over the center of a division ring, Proc. Amer. Math. Soc. 93 (1985), 590-592. MR 86g:16026

10. L. Makar-Limanov, On subnormal subgroups of skew fields, J. Algebra 114 (1988), 261-267. MR 89d:16024

11. $\ldots$, On subalgebras of the first Weyl algebra, Comm. Algebra 19 (1991), 1971-1982. MR 92g:16049

12. L. Makar-Limanov and P. Malcolmson, Free subalgebras of enveloping algebras, Proc. Amer. Math. Soc. 11 (1991), 315-322. MR 91f:16023

Department of Mathematics, University of Sao Paulo, CP 66281, 05389-970 Sao Paulo SP, BRAZIL

E-mail address: jzg@ime.usp.br

Department of Mathematics, University of Alberta, Edmonton, Alberta, Canada T6G 2G1

E-mail address: mazi@schur.math.ualberta.ca 